IP-ASTP-18-92

\title{
QCD Sum Rule and Perturbative QCD Approaches to Pion Compton Scattering
}

\author{
Claudio Corianò* and Hsiang-nan $\mathrm{Li}^{\dagger}$ \\ *Institute for Theoretical Physics, University of Stockholm \\ S 113 Vanadisvagen, Stockholm, Sweden \\ and \\ Dipartimento di Fisica, Universita' degli Studi di Parma \\ and INFN Gruppo Collegato di Parma, Italy \\ †Institute of Physics, Academia Sinica, \\ Taipei, Taiwan 11529, R.O.C.
}

July 10, 2018

\begin{abstract}
We compare two approaches to the description of pion Compton scattering at moderate momentum transfer, one being based on local duality QCD sum rules for the invariant amplitudes of the process, which have been derived recently, and the other on the modified factorization formula with Sudakov effects included. We find that perturbative QCD predictions are dominant over those from QCD sum rules only for the scattering angle greater than $90^{\circ}$.
\end{abstract}


1. Introduction. The method of QCD sum rules [1] has been very successful in describing many of the static and dynamical properties of hadrons at intermediate energy, a regime where non-perturbative effects are important. Based on this semi-phenomenological approach, important information on the quark distribution functions of hadrons [2-4] and on their electromagnetic form factors at momentum transfer $Q$ about 1 to $2 \mathrm{GeV}$ has been obtained [57]. On the other hand, the factorization formula, with Sudakov suppression taken into account for soft gluon exchange, has enlarged the applicability of perturbative QCD (pQCD) to elastic hadron form factors down to $Q \sim 2-3$ $\mathrm{GeV}$, and produces reliable results in agreement with experimental data [8]. The above energy scales indicate the transition from non-pQCD to pQCD at $Q^{2}$ around $4 \mathrm{GeV}^{2}$.

Then, natural questions to ask are whether these two approaches can be generalized to other more complicated processes, and on what energy scale they give comparable predictions. Recently, progress has been made in extending the QCD sum rule analysis to a four-point amplitude in pion Compton scattering $[9,10]$. A crossed version of Compton scattering, $p \bar{p} \rightarrow \gamma \gamma$, has been investigated based on the modified factorization formula [11]. In this letter we will study pion Compton scattering following the same approach, and compare the results with those from QCD sum rules. Their behavior at moderate momentum transfer may give hints about the above questions. Besides, the additional angular dependence of Compton scattering, which is absent in elastic form factors, will exhibit more information on the transition to $\mathrm{pQCD}$. Hence, Compton scattering provides a nontrivial comparision between the two methods, and experiments will determine which one gives a better description of the process.

In the sum rule approach, the lowest-order diagrams for pion Compton scattering are those without virtual gluons, as shown in fig. 1, which give real contributions $[9,10]$. The basic diagrams considered in pQCD have one extra exchanged gluon as in fig. 2. An imaginary contribution appears when the exchanged gluons in fig. $2 \mathrm{~d}$ and e (with two photons attaching to different quark lines) go on-shell, and thus brings in a phase [12], which distinguishes the lowest-order sum rule and pQCD approaches. Since the singularity due to this on-shell gluon is not removed by pion wave functions as in the case of form factors [13], Sudakov corrections are more important here in the sense of improving the self-consistency of the perturbative formula. Therefore, our work can be regarded as an attempt to incorporate Sudakov effects into 
Compton scattering.

Since the main purpose is to compare the two methods, we do not compute the full cross section of the process, but concentrate on one of the invariant amplitudes contained in the scattering matrix element. New sum rules for the invariant amplitude have been derived in ref. [10]. A modified perturbative expression for the same invariant amplitude will be given below. We study the process in a "brick-wall" frame, where the incoming pion momentum $p_{1}$ and outgoing one $p_{2}$ are assumed to have large "+" and "-" components respectively, and $\mathbf{p}_{T_{1}}=\mathbf{p}_{T_{2}}=0$. The incoming and outgoing photons, with momenta $q_{1}$ and $q_{2}$ respectively, are on-shell $\left(q_{1}^{2}=q_{2}^{2}=0\right)$. The Mandelstam invariants $s=\left(p_{1}+q_{1}\right)^{2}, t=\left(p_{1}-p_{2}\right)^{2}=-Q^{2}$ and $u=\left(p_{1}-q_{2}\right)^{2}$ are restricted in the physical region of $s>0, t<0$, and $u<0$.

To simplify the analysis, we assume that $s$ is larger than $-t$, but not so large that higher-order corrections proportional to $\ln (-t / s)$ diverge. It then suffices to consider the contributions which are not suppressed by $s$ or $u$. The ratio $-t / s$ characterizes the scattering angle $\theta$ of the photon, $\sin ^{2}(\theta / 2)=-t / s$. The condition $-t / s \rightarrow 0$ corresponds to forward scattering of the photon, while $-t / s \rightarrow 1$ to backward scattering. It is observed that the transition scale, on which the perturbative contributions become comparable to non-perturbative contributions, moves to large $-t$ as $\theta$ decreases. For forward scattering $(\theta \rightarrow 0)$ non-perturbative contributions always dominate. Only for $-t / s>0.5\left(\theta>90^{\circ}\right)$ are pQCD predictions important at moderate momentum transfer.

2. QCD Sum Rule Approach. A detailed QCD sum rule analysis of pion Compton scattering has been developed in $[9,10]$. In this section we will emphasize the essential aspects of the analysis and quote the results. We start with the correlator of 4-currents

$$
\begin{aligned}
\Gamma_{\alpha \mu \nu \beta}\left(p_{1}^{2}, p_{2}^{2}, s, t\right)= & i \int \mathrm{d}^{4} x \mathrm{~d}^{4} y \mathrm{~d}^{4} z \exp \left(-i p_{1} \cdot x+i p_{2} \cdot y-i q_{1} \cdot z\right) \\
& \times\left\langle 0\left|T\left(\eta_{\beta}(y) J_{\nu}(0) J_{\mu}(z) \eta_{\alpha}^{\dagger}(x)\right)\right| 0\right\rangle,
\end{aligned}
$$

where

$$
J_{\mu}=e_{u} \bar{u} \gamma_{\mu} u+e_{d} \bar{d} \gamma_{\mu} d, \quad \eta_{\alpha}=\bar{u} \gamma_{5} \gamma_{\alpha} d
$$

are the electromagnetic and axial currents respectively in terms of quark 
fields $u, d$ and charges $e_{u}=2 / 3, e_{d}=-1 / 3$. The pions are assumed to be off-shell $p_{1}^{2}=s_{1} \neq 0, p_{2}^{2}=s_{2} \neq 0$ with $s_{1}$ and $s_{2}$ their virtualities.

$\Gamma_{\alpha \mu \nu \beta}$ can be expressed in terms of many structures, each of which is associated with an invariant amplitude. We study the invariant ampltudes $H_{1}$ and $H_{2}$ with the structure proportional to $p_{1 \alpha} p_{2 \beta}[10]$

$$
\Gamma_{\alpha \mu \nu \beta} \propto f_{\pi}^{2} p_{1 \alpha} p_{2 \beta}\left(H_{1} e_{\mu}^{(1)} e_{\nu}^{(1)}+H_{2} e_{\mu}^{(2)} e_{\nu}^{(2)}\right)+\ldots
$$

where the helicity vectors $e^{(1)}$ and $e^{(2)}$ are defined by

$$
e^{(1) \mu}=\frac{N^{\mu}}{\sqrt{-N^{2}}}, \quad e^{(2) \mu}=\frac{P^{\mu}}{\sqrt{-P^{2}}}
$$

with

$$
\begin{aligned}
& N^{\mu}=\epsilon^{\mu \lambda \sigma \rho} P_{\lambda} q_{\sigma} K_{\rho}, \\
& P^{\mu}=p_{1}^{\mu}+\nu p_{2}^{\mu}-K^{\mu} \frac{p_{1} \cdot K+\nu p_{2} \cdot K}{K^{2}} \\
& \nu=\frac{p_{1} \cdot p_{2}-s_{1}}{p_{1} \cdot p_{2}-s_{2}} \\
& K=q_{1}+q_{2}, \quad q=q_{2}-q_{1} .
\end{aligned}
$$

The structure $p_{1 \alpha} p_{2 \beta}$ comes from the insertion of pion states into the correlator in eq. (1) and the substitution

$$
\begin{aligned}
& \left\langle\pi\left(p_{1}\right)\left|\eta_{\alpha}^{\dagger}(0)\right| 0\right\rangle=-i f_{\pi} p_{1 \alpha} \\
& \left\langle 0\left|\eta_{\beta}(0)\right| \pi\left(p_{2}\right)\right\rangle=i f_{\pi} p_{2 \beta}
\end{aligned}
$$

which interpolates between the vacuum and single pion state. We concentrate on the combination $H=H_{1}+H_{2}$, which is extracted by contracting $\Gamma_{\alpha \mu \nu \beta}$ with $-g^{\mu \nu} n^{\alpha} n^{\beta}$, where the circular polarization

$$
n^{\mu}=\left(e^{(2)} \pm i e^{(1)}\right)^{\mu}
$$

satisfies the conditions

$$
n \cdot q_{1}=n \cdot\left(p_{1}-p_{2}\right)=n^{2}=0 .
$$

These conditions, in full analogy with those in the case of form factors [7], are crucial for obtaining the correct asymptotic behavior of the sum rules, which 
is consistent with quark counting rules $[14,15]$. Note that the expression for $e^{(2)}$ given in eq. (4) is slightly different from that in [16]; the vector $P^{\mu}$ is defined including dependence on the extra factor $\nu$ due to the off-shell pions. This modified definition approaches the standard one given by $\nu=1$ at the pion pole, and guarantees the existence of $n^{\mu}$ with the requirements of eq. (8).

To evaluate the helicity form factor $H(s, t)$, it is simplest to consider its spectral function $\Delta\left(s_{1}, s_{2}, s, t\right)$ which is defined by the double discontinuity of $H$ on the cuts $0 \leq s_{1}, s_{2} \leq \lambda^{2} \approx(s+t) / 4$. This choice of $\lambda$ is to avoid the inclusion of $u$-channel resonances. The sum rule for $H(s, t)$, which is closely related to the finite energy sum rule in [17], has been formulated using the argument of analyticity for the correlator in eq. (1) in the finite region of the $s_{1}$ and $s_{2}$ complex planes [10]. The explicit form of $\Delta$ is essential both in the derivation of the sum rule and in the calculation of power corrections [18]. We will evaluate $\Delta$ using the standard cutting rules as developed in [5]. It is impossible to render all internal lines on-shell because of the off-shell assumption for the external pions. Only the case with all lines on-shell except the upper line contributes. We refer to [10] for a comprehensive derivation for the sum rule, and quote the final expression here

$$
\begin{aligned}
f_{\pi}^{2} H(s, t)= & \int_{0}^{s_{0}} \mathrm{~d} s_{1} \int_{0}^{s_{0}} \mathrm{~d} s_{2} \rho\left(s_{1}, s_{2}, s, t\right) \exp \left(-\frac{s_{1}+s_{2}}{M^{2}}\right) \\
& +c_{1} \frac{\left\langle 0\left|G_{\mu \nu} G^{\mu \nu}\right| 0\right\rangle}{M^{4}}+c_{2} \frac{\left\langle 0\left|(\bar{q} q)^{2}\right| 0\right\rangle}{M^{6}},
\end{aligned}
$$

$f_{\pi}=133 \mathrm{MeV}$ being the pion decay constant. The spectral density $\rho$ is given by

$$
\begin{aligned}
\rho\left(s_{1}, s_{2}, s, t\right) & =\frac{1}{\left(n \cdot p_{1}\right)\left(n \cdot p_{2}\right)} \Delta\left(s_{1}, s_{2}, s, t\right) \\
& \approx \frac{10}{3(2 \pi)^{2} \delta^{3}}\left[\left(s_{1}-s_{2}\right)^{2}-t\left(s_{1}+s_{2}\right)\right]\left(\frac{2 s-\delta}{s}+\frac{2 u-\delta}{u}\right) \\
\delta & =\sqrt{\left(s_{1}+s_{2}-t\right)^{2}-4 s_{1} s_{2}} .
\end{aligned}
$$

Those terms suppressed by extra powers of $t$ or $s$ have been neglected. The gluon and quark condensates $\left\langle 0\left|G_{\mu \nu} G^{\mu \nu}\right| 0\right\rangle$ and $\left\langle 0\left|(\bar{q} q)^{2}\right| 0\right\rangle$, arising from the operator product expansion of the correlator in eq. (1), give the power corrections which are suppressed by factors $\left(1 / M^{2}\right)^{n}, M^{2}$ being the Borel mass. 
The coefficients $c_{1}$ and $c_{2}$ can be obtained through the operator product expansion. The exact value of the quark-hadron duality interval $s_{0}$ is determined by demanding that the right-hand side of eq. (9) have the weakest sensitivity to the variation of $M^{2}$ [2-6]. Once $s_{0}$ is specified, the large $M^{2}$ limit is applied to diminish the power corrections, and the final expression for $H$ is written as a local duality approximation

$$
f_{\pi}^{2} H(s, t)=\int_{0}^{s_{0}} \mathrm{~d} s_{1} \int_{0}^{s_{0}} \mathrm{~d} s_{2} \rho\left(s_{1}, s_{2}, s, t\right),
$$

which behaves like $1 / Q^{4}$ asymptotically. We will address the calculation of the power corrections in a seperate work [18], and assign an approximate value to $s_{0}$ here. It is also possible to derive a similar sum rule for the single invariant amplitude $H_{1}$.

3. Perturbative $Q C D$ approach. Part of the basic diagrams for the perturbative QCD approach are shown in fig. 2. Each represents a class of diagrams which can be transformed to each other by permuting the incoming and outgoing pions, or the two quark lines. They differ from fig. 1 by an extra exchanged gluon. We will incorporate Sudakov effects for soft gluon exchange, the case in which the running coupling constant $\alpha_{s}$ with its argument set to the gluon energy is large and lowest-order PQCD does not make sense. The method to calculate pion Compton scattering based on these diagrams is similar to that developed for electromagnetic form factors [8]. Following the same reasoning and procedures, the modified factorization formula for $H(s, t)$ is given by

$$
\begin{aligned}
H(s, t)= & \sum_{l=1}^{2} \int_{0}^{1} \mathrm{~d} x_{1} \mathrm{~d} x_{2} \mathrm{~d}^{2} \mathbf{k}_{T_{1}} \mathrm{~d}^{2} \mathbf{k}_{T_{2}} \psi\left(x_{2}, \mathbf{k}_{T_{2}}, p_{2}\right) \\
& \times T_{H_{l}}\left(x_{i}, s, t, \mathbf{k}_{T_{i}}\right) \psi\left(x_{1}, \mathbf{k}_{T_{1}}, p_{1}\right) .
\end{aligned}
$$

The additional dependence on transverse momentum $\mathbf{k}_{T}$ carried by a valence quark has been included into the pion wave function $\psi$ and the hardscattering kernel $T_{H}$. Eq. (12) can be understood as an intermediate step in the standard factorization program [19], where $\mathbf{k}_{T}$ in $T_{H}$ is assumed to give higher-power $\left(\mathbf{k}_{T}^{2} / Q^{2}\right)$ correction and thus ignored. In fact, this approximation is not proper when the exchanged gluon becomes soft. The contributions to the hard scatering from each diagram in fig. 2, obtained by contracting 
the two photon vertices with $-g^{\mu \nu}$, are given in table. 1 . The transverse momenta on the virtual quark lines have been neglected in the derivation since they are associated with linear, instead of quadratic, divergences in $x_{i}$, and hence less important than that on the gluon line [8]. The contributions from fig. $2 \mathrm{~d}$ and e, like fig. 1c, are suppressed by $s$ as stated in the previous section. All contributions are grouped into only two terms $(l=1,2)$ using the permutative symmetry.

Rewriting eq. (12) in terms of the Fourier transformed functions, and inserting the large- $b$ asymptotic behavior of the wave function [19], we have

$$
\begin{aligned}
H(s, t)= & \sum_{l=1}^{2} \int_{0}^{1} \mathrm{~d} x_{1} \mathrm{~d} x_{2} \phi\left(x_{1}\right) \phi\left(x_{2}\right) \int_{0}^{\infty} b \mathrm{~d} b \tilde{T}_{H_{l}}\left(x_{i}, s, t, b, w_{l}\right) \\
& \times \exp \left[-S\left(x_{i}, b, Q, w_{l}\right)\right] .
\end{aligned}
$$

where $b$, introduced by Fourier transformation, is the seperation between the two valence quark lines. Note the extra Sudakov factor $\exp (-S)$ compared to the standard factorization formula, which arises from an all-order summation of the collinear enhancements of radiative corrections to fig. 2. The exponent $S$ is written as [8]

$$
S\left(x_{1}, x_{2}, b, Q, w\right)=\sum_{i=1}^{2}\left(s\left(x_{i}, b, Q\right)+s\left(1-x_{i}, b, Q\right)\right)-\frac{2}{\beta_{1}} \ln \frac{\hat{w}}{-\hat{b}},
$$

with

$$
\begin{aligned}
s(\xi, b, Q)= & \frac{A^{(1)}}{2 \beta_{1}} \hat{q} \ln \left(\frac{\hat{q}}{-\hat{b}}\right)+\frac{A^{(2)}}{4 \beta_{1}^{2}}\left(\frac{\hat{q}}{-\hat{b}}-1\right)-\frac{A^{(1)}}{2 \beta_{1}}(\hat{q}+\hat{b}) \\
& -\frac{A^{(1)} \beta_{2}}{16 \beta_{1}^{3}} \hat{q}\left[\frac{\ln (-2 \hat{b})+1}{-\hat{b}}-\frac{\ln (2 \hat{q})+1}{\hat{q}}\right] \\
& -\left[\frac{A^{(2)}}{4 \beta_{1}^{2}}-\frac{A^{(1)}}{4 \beta_{1}} \ln \left(\frac{e^{2 \gamma-1}}{2}\right)\right] \ln \left(\frac{\hat{q}}{-\hat{b}}\right) \\
& -\frac{A^{(1)} \beta_{2}}{32 \beta_{1}^{3}}\left[\ln ^{2}(2 \hat{q})-\ln ^{2}(-2 \hat{b})\right] .
\end{aligned}
$$

The variables $\hat{q}, \hat{b}$ and $\hat{w}$ are defined by

$$
\begin{aligned}
\hat{q} & \equiv \ln [\xi Q /(\sqrt{2} \Lambda)] \\
\hat{b} & \equiv \ln (b \Lambda) \\
\hat{w} & \equiv \ln (w / \Lambda)
\end{aligned}
$$


where the scale parameter $\Lambda \equiv \Lambda_{\mathrm{QCD}}$ will be set to $0.1 \mathrm{GeV}$. The coefficients $\beta_{i}$ and $A^{(i)}$ are

$$
\begin{aligned}
& \beta_{1}=\frac{33-2 n_{f}}{12}, \quad \beta_{2}=\frac{153-19 n_{f}}{24}, \\
& A^{(1)}=\frac{4}{3}, \quad A^{(2)}=\frac{67}{9}-\frac{\pi^{2}}{3}-\frac{10}{27} n_{f}+\frac{8}{3} \beta_{1} \ln \left(\frac{e^{\gamma}}{2}\right)
\end{aligned}
$$

with $n_{f}=3$ the number of quark flavors and $\gamma$ the Euler constant. The Sudakov factor is always less than 1 as explained in ref. [8], and decreases quickly in the large- $b$ region. The function $\phi$, obtained by factoring the $Q$ and $b$ dependences from the transformed wave function into Sudakov logarithms, is taken as the Chernyak and Zhitnitsky model [2]

$$
\phi^{C Z}(x)=\frac{15 f_{\pi}}{\sqrt{2 N_{c}}} x(1-x)(1-2 x)^{2}
$$

where $N_{c}=3$ is the number of colors.

The transformed hard scatterings $\tilde{T}_{H_{l}}$ are given by

$$
\begin{aligned}
\tilde{T}_{H_{1}}= & \frac{16 \pi \mathcal{C}_{F}\left(e_{u}^{2}+e_{d}^{2}\right) \alpha_{s}\left(w_{1}\right)}{\left(1-x_{1}\right)\left(1-x_{2}\right)} K_{0}\left(\sqrt{\left|r_{1}\right|} b\right)\left(\frac{\left[\left(1-x_{1}\right) t+u\right]\left[\left(1-x_{2}\right) t+u\right]}{s^{2}}\right. \\
& \left.+\frac{\left[\left(1-x_{1}\right) t+s\right]\left[\left(1-x_{2}\right) t+s\right]}{u^{2}}-4\left(1-x_{2}\right)\right)
\end{aligned}
$$

from the classes of fig. 1a-c, and

$$
\begin{aligned}
\tilde{T}_{H_{2}}= & 32 \pi \mathcal{C}_{F} e_{u} e_{d} \alpha_{s}\left(w_{2}\right)\left[\theta\left(-r_{2}\right) K_{0}\left(\sqrt{\left|r_{2}\right|} b\right)-\theta\left(r_{2}\right) \frac{i \pi}{2} H_{0}^{(1)}\left(\sqrt{r_{2}} b\right)\right] \\
& \times\left(\frac{1}{x_{1}\left(1-x_{1}\right)}-\frac{\left(1+x_{2}-x_{1} x_{2}\right) t^{2}+\left(1+x_{2}-x_{1}\right) u t}{x_{2}\left(1-x_{1}\right) s^{2}}\right. \\
& \left.+\frac{1}{x_{2}\left(1-x_{2}\right)}-\frac{\left(1+x_{1}-x_{1} x_{2}\right) t^{2}+\left(1+x_{1}-x_{2}\right) s t}{x_{1}\left(1-x_{2}\right) u^{2}}\right)
\end{aligned}
$$

from the class fig. 1d-e with

$$
r_{1}=x_{1} x_{2} t, \quad r_{2}=x_{1} x_{2} t+x_{1} u+x_{2} s
$$


$K_{0}$ and $H_{0}^{(1)}$ in eqs. (19) and (20) are the Bessel functions in the standard notation. The imaginary contribution comes from eq. (20). The argument $w_{l}$ of $\alpha_{s}$ is defined by the largest mass scale in the hard scattering,

$$
w_{1}=\max \left(\sqrt{\left|r_{1}\right|}, \frac{1}{b}\right), \quad w_{2}=\max \left(\sqrt{\left|r_{2}\right|}, \frac{1}{b}\right) .
$$

As long as $b$ is small, soft $r_{l}$ does not lead to large $\alpha_{s}$. Therefore, the nonperturbative region in the modified factorization is characterized by large- $b$, the region which is suppressed by Sudakov effects. Eq. (13), as a perturbative expression, is thus relatively self-consistent compared to the leading-power factorization [20].

4. Numerical Analysis. To evaluate the invariant amplitude $H(s, t)$ from the sum rule, the value of $s_{0}$ must be determined through a stability analysis on the $M^{2}$ dependence of eq. (9) as in [2-6]. However, we know that $s_{0}$ must take a value between the masses of pion $\left(m_{\pi}^{2}=0\right)$ and $\mathrm{A}_{1}$-meson $\left(m_{A_{1}}^{2}=\right.$ $1.2 \mathrm{GeV}^{2}$ ). Hence, we estimate that $s_{0}$ resides in the reasonable range $0.6<s_{0}<0.8 \mathrm{GeV}^{2}$, as referred to the case of pion form factor. $H(s, t)$ with $s_{0}=0.6,0.7$ and $0.8 \mathrm{GeV}^{2}$ are computed and found to vary slowly in the above range of $s_{0}$ for $Q^{2}>4 \mathrm{GeV}^{2}$. Therefore, $s_{0}$ will be set to $0.7 \mathrm{GeV}^{2}$ in eq. (11) for the sum rule analysis. Results of $Q^{2} H$ for different $-t / s$ are shown in fig. 3 with $4<Q^{2}<25 \mathrm{GeV}^{2}$. It is observed that $H$ decreases monotonously following the $1 / Q^{4}$ asymptotical behavior, and has a weak angular dependence.

Results derived from the modified expression eq. (13) in the same range of $Q^{2}$ are also shown in fig. 3, where $|H|$ denotes the magnitude of $H$. It is found that $|H|$ has only little (logarithmical) deviation from the expected $1 / Q^{2}$ behavior, and drops quickly with decreasing $-t / s$. That is, $\mathrm{pQCD}$ predictions have a stronger angular dependence. The curve from eq.(13) denoted by $-t / s=0.6\left(\theta=100^{\circ}\right)$ is always above the corresponding curve derived from the sum rule for $4<Q^{2}<25 \mathrm{GeV}^{2}$. It indicates the dominance of perturbative contribution in large-angle Compton scattering. For $-t / s=0.5$ $\left(\theta=90^{\circ}\right)$ sum rule and pQCD predictions are comparable, and the transition scale is about $6 \mathrm{GeV}^{2}$. As $-t / s$ drops, the transition scale increases, and the non-perturbative contribution becomes dominant when $-t / s$ goes down to $0.2\left(\theta=50^{\circ}\right)$. Based on the above analysis, we conjecture that the present pQCD calculation for proton Compton scattering [20] or its crossed version 
like $p \bar{p} \rightarrow \gamma \gamma[11]$ should be complemented by the QCD sum rule description at small angles.

The phase $\phi$ of $H$ obtained from eq. (13) is also studied, and its $Q^{2}$ dependence for different $-t / s$ is shown in fig. 4 . It is found that $\phi$ is very stable at intermediate angles, but changes drastically at small angles.

The corresponding results from the standard factorization formula with $\mathbf{k}_{T}$ neglected in $T_{H}$ and $\alpha_{s}=0.3$ in eq. (12) are exhibited for reference, and are similar to those from the modified one. This choice of $\alpha_{s}$ has been used in order to fit experimental data from proton Compton scattering [20]. However, $|H|$ shows an exact $1 / Q^{2}$ behavior and $\phi$ is independent of $Q^{2}$ at a fixed angle. Note the difference between the standard and modified factorizations that $\alpha_{s}$ is effectively regarded as a free parameter in the former case.

5. Conclusion. In this letter we have shown that the extension of both the QCD sum rule method and modified factorization formula to pion Compton scattering is possible, because their agreement at intermediate scattering angles is explicit. We believe that this coincidence is not trivial due to the very different theoretical bases of the two approaches. The transition scale from QCD sum rules to pQCD is found to vary with scattering angles. QCD sum rules give important contribution at small angles, and pQCD becomes dominant only for $\theta>90^{\circ}$ from our analysis. Our results have suggested a new and interesting interplay between factorization theorems and sum rule methods in Compton scattering. All these observations need to be compared with experiments. To examine the conclusions more exactly, it is necessary to determine a precise value for $s_{0}$, and thus the calculation for gluon and quark condensates, along with a detailed discussion on the stability of the sum rule, is involved [18]. It is also of interest to generalize the two methods to more complicated case like proton Compton scattering [20], for which experimental data are available.

We thank Prof. G. Sterman for suggesting this investigation, and Profs. T.H. Hansson and H. Rubinstein for clarifying discussions. C. C. thanks Prof. G. Marchesini and Dr. F. Fiorani for stimulating conversations and for their hospitality at the Physics Department of Parma University, and Profs. N. Isgur and A.V. Radyushkin for their hospitality at CEBAF. This work was supported in part by the National Science Council of R.O.C. under Grant No. NSC82-0112-C001-017 and by INFN of Italy. 


\section{References}

[1] M.A. Shifman, A. I. Vainshtein and V.I. Zakharov, Nucl. Phys. B147 (1979) 385, 448, 519.

[2] V.L. Chernyak and A.R. Zhitnitsky, Nucl. Phys. B201 (1982) 492; Phys. Rep. 112 (1984) 173.

[3] I.D. King and C.T. Sachrajda, Nucl. Phys. B279 (1987) 785.

[4] M. Gari and N.G. Stefanis, Phys. Lett. B175 (1986) 462.

[5] B.L. Ioffe and A.V. Smilga, Phys. Lett. B114 (1982) 353; Nucl. Phys. B216 (1983) 373.

[6] A.V. Radyushkin, Acta Phys. Polon. B15 (1984) 403.

[7] V.A. Nesterenko and A.V. Radyushkin, Phys. Lett. B115 (1982) 410; Sov. Phys.-JETP Lett. 35.

[8] H.N. Li and G. Sterman, Nucl. Phys. B381 (1992) 129; H.N. Li, Stony Brook preprint ITP-SB-92-25.

[9] C. Corianò, Ph.D. thesis, Stony Brook (1992) unpublished.

[10] C. Corianò, A. Radyushkin and G. Sterman, Stony Brook preprint ITPSB-92-70 (CEBAF preprint TH-92-32).

[11] T. Hyer, SLAC preprint SLAC-PUB-5889.

[12] G.R. Farrar, G. Sterman and H. Zhang, Phys. Rev. Lett. 62 (1989) 2229.

[13] E. Maina and G.R. Farrar, Phys. Lett. B206 (1988) 120.

[14] V.A. Matveev, R.M. Muradyan and A.N. Tavkhelidze, Lett. Nuovo Cimento 7 (1973) 719 .

[15] S.J. Brodsky and G.R. Farrar, Phys. Rev. Lett. 31 (1973) 1153.

[16] V.B. Beretetskii, E.M. Lifshitz and L.P. Pitaevskii, Relativistic Quantum Theory, (Pergamon Press, 1971). 
[17] A.A. Logunov, L.D. Soloviev and A.N. Tasvkelidze, Phys. Lett. B24 (1967) 181.

[18] C. Corianò and H.N. Li, in preparation.

[19] J. Botts and G. Sterman, Nucl. Phys. B325 (1989) 62.

[20] G.R. Farrar and H. Zhang, Phys. Rev. D41 (1990) 3348; 42 (1990) 2413(E); A.S. Kronfeld and B. Nižić, Phys. Rev. D44 (1991) 3445. 
Table 1. The expressions of the hard scattering $T_{H}$ corresponding to the diagrams in fig. 2.

\begin{tabular}{cc}
\hline \hline Diagram & $T_{H} /\left(64 \pi \alpha_{s}\right)$ \\
\hline (a) & $\frac{-e_{u}^{2}\left[\left(1-x_{1}\right) t+u\right]\left[\left(1-x_{2}\right) t+u\right]}{\left(1-x_{1}\right)\left(1-x_{2}\right) s^{2}\left[x_{1} x_{2} t-\left(\mathbf{k}_{T_{1}}-\mathbf{k}_{T_{2}}\right)^{2}\right]}$ \\
(b) & $\frac{e_{u}^{2}}{\left(1-x_{1}\right)\left[x_{1} x_{2} t-\left(\mathbf{k}_{T_{1}}-\mathbf{k}_{T_{2}}\right)^{2}\right]}$ \\
(c) & $\frac{e_{u}^{2}}{\left(1-x_{1}\right)\left[x_{1} x_{2} t-\left(\mathbf{k}_{T_{1}}-\mathbf{k}_{T_{2}}\right)^{2}\right]}$ \\
(d) & $\frac{-e_{u} e_{d}}{x_{1}\left(1-x_{1}\right)\left[x_{1} x_{2} t+x_{1} u+x_{2} s-\left(\mathbf{k}_{T_{1}}-\mathbf{k}_{T_{2}}\right)^{2}\right]}$ \\
(e) & $\frac{e_{u} e_{d}\left[\left(1+x_{2}-x_{1} x_{2}\right) t^{2}+\left(1+x_{2}-x_{1}\right) u t\right]}{x_{2}\left(1-x_{1}\right) s^{2}\left[x_{1} x_{2} t+x_{1} u+x_{2} s-\left(\mathbf{k}_{T_{1}}-\mathbf{k}_{T_{2}}\right)^{2}\right]}$ \\
\hline \hline
\end{tabular}




\section{Figure Captions}

Fig. 1. Diagrams for the pion Comptom scattering in the QCD sum rule analysis.

Fig. 2. Part of the diagrams for the pion Comptom scattering in the perturbative QCD analysis.

Fig. 3. Dependence of $Q^{2}|H(s, t)|$ on $Q^{2}$ derived from QCD sum rules (dashed lines), from the modified factorization (solid lines) and from the leading-power factorization (dotted lines) for $(\mathrm{a})-t / s=0.6\left(\theta=100^{\circ}\right)$, (b) $-t / s=0.5\left(\theta=90^{\circ}\right)$ and $(\mathrm{c})-t / s=0.2\left(\theta=50^{\circ}\right)$.

Fig. 4. Dependence of the phase $\phi$ on $Q^{2}$ from the modified factorization (solid lines) and from the leading-power factorization (dotted lines) for (a) $-t / s=0.6,(\mathrm{~b})-t / s=0.5,(\mathrm{c})-t / s=0.2$ and $(\mathrm{d})-t / s=0.02$. 